\title{
Deep Learning: The Impact on Future eLearning
}

\author{
https://doi.org/10.3991/ijet.v15i01.11435 \\ Anandhavalli Muniasamy ${ }^{(凶)}$, Areej Alasiry \\ King Khalid University, Abha, Saudi Arabia \\ anandhavalli.dr@gmail.com
}

\begin{abstract}
Learning as technology becomes more affordable in higher education but having a big barrier in the cost of developing its resources. Deep learning using artificial intelligence continues to become more and more popular and having impacts on many areas of eLearning. It offers online learners of the future with intuitive algorithms and automated delivery of eLearning content through modern LMS platforms. This paper aims to survey various applications of deep learning approaches for developing the resources of the eLearning platform, in which predictions, algorithms, and analytics come together to create more personalized future eLearning experiences. In addition, deep learning models for developing the contents of the eLearning platform, deep learning framework that enable deep learning systems into eLearning and its development, benefits \& future trends of deep learning in eLearning, the relevant deep learning based artificial intelligence tools and a platform enabling the developer and learners to quickly reuse resources are clearly summarized. Thus, deep learning has evolved into developing ways to repurpose existing resources can mitigate the expense of content development of future eLearning.
\end{abstract}

Keywords - eLearning, Deep Learning (DL), Learning Management System (LMS), Artificial Intelligence, Machine Learning.

\section{Introduction}

Due to the advent of information and communication technology (ICT) in the current era, there has emerged a multiplicity of applications in Higher Education (HE). In that context, eLearning was launched as a way of responding to the new set of educational demands. eLearning has been defined as learning management software systems that synthesize the functions of computer-mediated communications software and online methods of delivering course materials [7]. One of the most important reasons given for the large-scale investment in web-based technology is their potential to enhance teaching and learning [22], as well as to encourage the development of student-centered, independent learning [30] and to foster a deeper approach to learning [12]. According to a survey conducted by [9], eLearning content development for one hour varied from 49 to 125 hours. Although the advent of eLearning environment promised more flexible and independent learning due to its scalability, it can still pose a barrier to institutions that can't afford the initial investment. So, developing ways to repurpose existing eLearning resources can mitigate the expense of content development.

As we progress in the field of AI, new techniques such as deep learning and artificial neural network are being developed to improve the effectiveness of machine learning and making the applications of AI far-reaching and meaningful. Machine 
learning tries to model the world, but deep learning attempts to model the human brain to create and maintain its own representations of the world. Deep learning involves algorithms that predict possible outcomes based on user data, which allow a computer to display behaviors learned from experiences, rather than human interactions. It enables automation using algorithms to learn from data and make determinations and predictions. Every new information that the deep learning model receives makes it more intuitive.

Due to massive information overload on the web, it's hard to index and reuse existing contents. Classifying contents according to domain-specific concept hierarchies could address the problem of indexing and reusability. As a result, automatic classifiers are in high demand due to the difficulties in manual classification. Deep learning can aid in eLearning developing by improving the classification of elements of content, as digital learners increasingly expect content to be offered in multiple formats and on a variety of platforms. In the eLearning domain, the deep learning process takes place autonomously, from extracting and evaluating the data sets from the LMS to predicting what online learners need to be based on their past performance.

This paper summarizes the impacts of deep learning for resource management in eLearning, as well as discuss how it will shape eLearning in the future. We briefly review four relevant aspects from learner and developer perspectives:

- Motivations of applying deep learning in eLearning

- Deep Learning Framework for developing the contents of the eLearning platform

- Tools and platforms that enable deep learning systems into eLearning and its development

- Benefits \& future trends of DL in eLearning

The rest of the paper is planned as follows: Section 2 focuses on a survey related to deep learning in eLearning. Section 3 describes deep learning techniques in the development of eLearning platform. Section 4 covers the benefits of DL in eLearning. Tools, platforms and Future trends of DL in eLearning is discussed in section 5 and section 6 concludes the summary.

\section{$2 \quad$ Literature Review}

Deep learning process data from the lower level to a higher level and gradually composing increasing semantic concepts, simulates the hierarchical structure of the human brain. Recently, deep learning is growing as the most popular tool for big data analysis [40] and artificial intelligence [40]. By using deep learning algorithm, artificial intelligence has a big breakthrough in many areas, such as face recognition [40], image processing [3], and speech recognition [2]. Inspire by those, an attempt has been made to survey the impact of deep learning in an eLearning environment. 


\section{1 eLearning}

eLearning is a type of delivery method used in distance education that allows the synchronous and asynchronous exchange of resources over a communication network [23]. It is a system surrounding the learner and the teacher in terms of technical and social aspects, which promote communication and collaboration among students and instructors [24]. Distance learning, at times called e-learning, is a formalized showing and learning framework particularly intended to be completed remotely by utilizing electronic correspondence [11]. Many higher institutions have been using course management software (e.g., Blackboard, LMS, WebCT, and Moodle) to provide eLearning that complement traditional classroom-based instruction. Authors in the literature [32] and [1] argue that eLearning offers a balance between the technology enablers and the acceptance issue. Rosenberg in [32] made available an important roadmap for keeping the e-learning in sustainable and continuous growth of showing twenty-year chronology of e-Learning evolution. Incorporating the recent technology Web 2.0 in the learning process is complex, but at the same time may shift the paradigm of learning and training, podcasts, weblogs, wikis and web sharing applications induced e-Learning 2.0 to emerge [16]. E-Learning 2.0 environment is more focus on engaging the students in the learning process.

The main difference between traditional machine learning and deep learning algorithms is in the feature engineering which requires domain expertise and a timeconsuming process (Fig.1). Deep learning algorithms involve automatic feature engineering, whereas we need to handcraft the features in traditional machine learning algorithms. Deep learning combines advances in computing power and neural networks with many layers (Fig. 2) to learn complicated patterns in large amounts of data. It is an extension of a classical neural network and uses more hidden layers so that the algorithms can handle complex data with various structures [14].

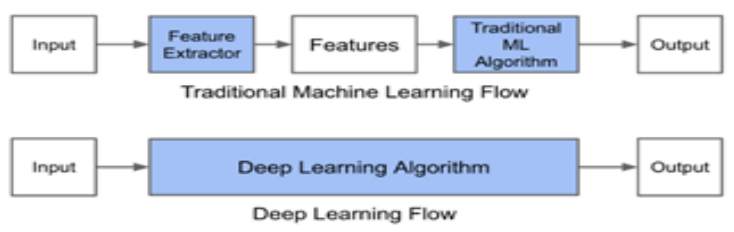

Fig. 1. Machine learning vs. Deep Learning

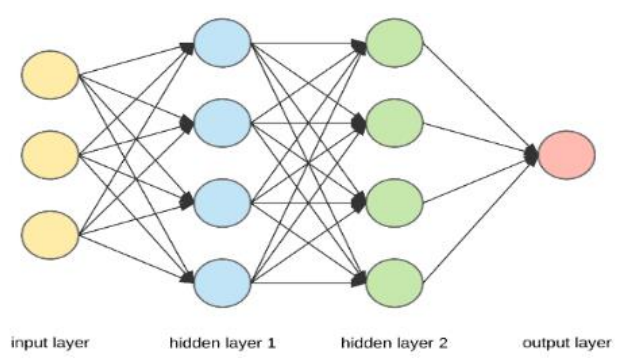

Fig. 2. Deep learning with two hidden layers. 
Deep learning includes four common algorithms that allow the system to predict future outcomes and detected patterns based on specific user data.

- Supervised learning algorithm uses past examples and new data sets to predict the outcomes. Initially, the system starts with provided inputs and outputs to train the software. Then, the system can automatically construct outputs or targets for new data sets over time.

- Unsupervised learning algorithms do not involve any labels or data classifications. The system evaluates data to identify patterns and make inferences or predictions.

- Semi-supervised learning algorithm combines unlabelled data and human-based training, in which labelled online resources are provided to map out certain inputs and outputs with greater accuracy.

- Reinforcement learning algorithm includes a specific task or goal that the system should complete. Throughout the process, it receives feedback to learn the desired behaviours for the most effective approach via reinforcement signals.

\subsection{Deep learning applications in eLearning}

Personalized learning path [10]: It is a learner-centered eLearning approach that emphasizes learner-specific goals and objectives, as well as preferences for mapping the courses. A sequence of courses or learning materials frames the learning path that allows learners to build their knowledge progressively. It is generated and changed dynamically based on learner's job roles, their area of interest, learners' progress, learning preferences, demographic information, competencies or knowledge levels, etc. Usually, a learner model is built at the back-end to identify, collect and update variables to personalize different content to each individual learner.

Chatbots [34]: It acts as the virtual assistant that provides conversational answers, serves as a quick reference guide, and as a knowledge management tool can tap into various sources of information that are distributed across the organization. An intelligent tutoring system presents a learning concept with a series of conversations for coaching and performance support.

Performance indicator [39]: It is used to pinpoint a certain learning pattern, such as significant changes in course failings, so instructors can advise the students before it is too late. It will also provide a more effective way to analyze learner engagement data and identify their patterns. Accordingly, the suggestion for the redesign of the content will be provided as additional support to learners who are failing to complete a course or a learning activity.

Virtual teaching assistant [35], introduced by Georgia Institute of Technology, online classes to answer questions with distinct and clear solutions. The students can ask the same kind of questions repeatedly which will help to increase their knowledge base in different contexts. 


\section{Deep Learning Framework for eLearning}

There are five widespread kinds of information [17]:

- Sequences

- Association

- Classifications

- Clusters

- Prediction

Similarly, the general purpose of deep learning model is to provide powerful solutions to associations, classifications, clusters and predictions problems. In the eLearning applications, the commonly used deep learning models include:

- Convolution neural network (CNN): The CNN was first proposed and applied method for the high-dimensional image analysis by [26]. It consists of convolutional filters, which transform 2D into 3D.

- Recurrent neural network (RNN): It is a neural net architecture with recurrent connections between hidden states and has the capability of learning sequences and model time dependencies also. The recurrent connections are used to detect relationships not only between inputs but also over time. Therefore, it is well suited to health problems that often involve modelling clinical data changes over time [41].

- Deep belief network (DBN): This model has a unidirectional connection at two layers on the top of layers. The hidden layers of each sub-network serve as a visible layer for the next layer.

- Deep neural network (DNN): It has more than two layers, which allows the complex non-linear relationship.

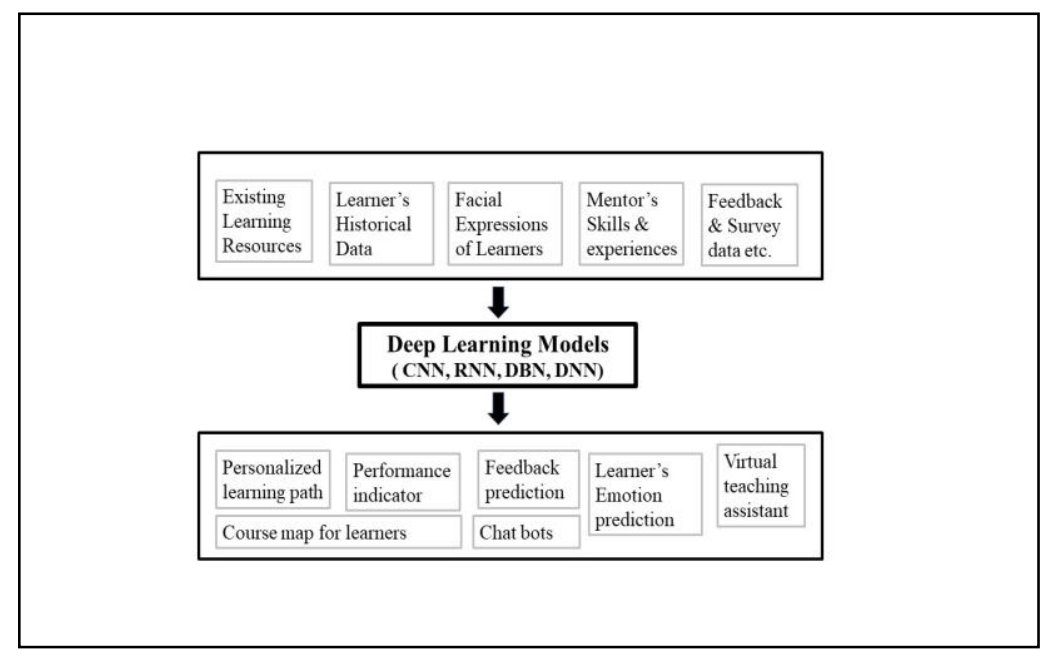

Fig. 3. Deep Learning Framework for the eLearning environment 
The framework (see Fig.2) shows the ways in which deep learning models can aid in eLearning development by using:

- Existing learning resources to produce the personalized learning path and classification of the contents based on learner's interest.

- Learner's historical data to predict their performance and associate course map for individual learners.

- Facial expressions of learners to predict their emotions in the eLearning environment.

- Mentor's skills and experience to support peer-to-peer interactions with the learners.

- Information, say, in a video, an infographic, the text file containing the video transcript, and a chat-based quiz that offers feedback.

Table 1 summarizes the recent applications deep learning in eLearning, technical advantages and limitations of each deep learning models.

Table 1. Deep Learning Models Summary in eLearning

\begin{tabular}{|l|l|l|l|}
\hline Model & \multicolumn{1}{|c|}{$\begin{array}{c}\text { Recent Applications in } \\
\text { eLearning }\end{array}$} & \multicolumn{1}{c|}{ Advantages } & \multicolumn{1}{c|}{ Limitations } \\
\hline CNN & $\begin{array}{l}\text { Predict the latent factors of the } \\
\text { learning resources. [36] } \\
\text { Using facial expression to detect } \\
\text { the emotions of students [37] }\end{array}$ & $\begin{array}{l}\text { Provide very good performance } \\
\text { for 2D data. Model learning is } \\
\text { fast }\end{array}$ & $\begin{array}{l}\text { Need lots of labeled data for } \\
\text { classification }\end{array}$ \\
\hline RNN & $\begin{array}{l}\text { Student Feedback Prediction } \\
\text { using Kinect [20] }\end{array}$ & $\begin{array}{l}\text { Learn sequential events and } \\
\text { model time dependencies. } \\
\text { Provide good accuracy in } \\
\text { speech \& character recognition } \\
\text { and NLP related tasks }\end{array}$ & $\begin{array}{l}\text { Need big datasets. } \\
\text { Has many issues due to gradient } \\
\text { vanishing }\end{array}$ \\
\hline DBN & $\begin{array}{l}\text { Natural language understanding } \\
\text { [33], Large-vocabulary speech } \\
\text { recognition [18], [25] }\end{array}$ & $\begin{array}{l}\text { Supports both supervised and } \\
\text { unsupervised learning model }\end{array}$ & $\begin{array}{l}\text { Initialization process makes the } \\
\text { training process computationally } \\
\text { expensive }\end{array}$ \\
\hline DNN & $\begin{array}{l}\text { Visual representational trans- } \\
\text { formations in distance learning } \\
\text { [29] }\end{array}$ & $\begin{array}{l}\text { Widely used with great accura- } \\
\text { cy }\end{array}$ & $\begin{array}{l}\text { Training process is not trivial as } \\
\text { the error propagated back to the } \\
\text { previous layers and becomes } \\
\text { very small } \\
\text { Learning process is also too } \\
\text { much slow }\end{array}$ \\
\hline
\end{tabular}

As digital learners increasingly expect content to be offered in multiple formats, many eLearning developments "repurposing" the existing resources and implementing "plus-one" design. A developer focus on the track in which the concept is mentioned and explained, then locate the content to reuse it. Alternatively, a deep learning tool can identify and efficiently locating suitable resources, thus enabling the developer to quickly reuse content. Automating this process enhances the consistency of learning resources and facilities across platforms while also saving development time. 


\section{Deep Learning Tools for eLearning Platform Development}

Table 2 shows the tools and platforms for deep learning tools in eLearning applications. The advent of available DL tools and components allows eLearning developers to purchase or license algorithms to avoid the time and expense of developing their own.

Table 2. Deep Learning Tools and Platforms in eLearning Applications

\begin{tabular}{|l|l|}
\hline \multicolumn{1}{|c|}{ Tools \& Platforms } & \multicolumn{1}{c|}{ Features } \\
\hline AIaaS (AI as a Service) [4] & Cloud based AI tools and algorithms for the eLearning development \\
\hline Microsoft Azure [27] & Cloud based AI applications, such as image recognition or bot-based apps \\
\hline IBM's Watson [21] & Cloud-based AI services to use with Watson platform \\
\hline Amazon Web Services [5] & Amazon's cloud-based AI services \\
\hline Google's Tensorflow [38] & $\begin{array}{l}\text { An open source artificial intelligence library, using data flow graphs to build } \\
\text { models and allows developers to create large-scale neural networks with many } \\
\text { layers }\end{array}$ \\
\hline Caffe [8] & A cross-platform support C++, Matlab, and Python programming interfaces \\
\hline MXNet [28] & $\begin{array}{l}\text { An open source software library for numerical computation using data flow } \\
\text { graphs and supports DL architectures CNN and RNN }\end{array}$ \\
\hline Theano & $\begin{array}{l}\text { Provides capabilities like symbolic API supports looping control (scan), which } \\
\text { makes implementing RNNs easy and efficient }\end{array}$ \\
\hline Keras & Theano based deep learning library \\
\hline ConvNet [13] & Matlab based convolutional neural network toolbox \\
\hline Deeplearning4j [15] & $\begin{array}{l}\text { An open-source, Apache 2.0-licensed distributed neural net library in Java and } \\
\text { Scala }\end{array}$ \\
\hline Apache Singa [6] & Open source library for deep learning \\
\hline
\end{tabular}

When considering using deep learning in the eLearning development, it's advisable to choose a platform carefully as each platform has its own strengths and weaknesses. It also depends on the needs of the developers and learners as well as the technical skills of the developers who will be binding these tools and services to create deep learning enhanced eLearning tools.

\section{Discussion on Future Trends of Deep Learning in eLearning}

There are a variety of benefits that deep learning can offer online learners of the future, as well as organizations who invest in modern eLearning platforms that feature intuitive algorithms and automated delivery of eLearning content. Here are just a few of the most notable advantages for discussion:

Deep learning delivers more personalized eLearning content as it predicts outcomes based on past performance and individual learning goals to provide specific eLearning content in a personalized format. For example, it may skip several eLearning modules for more advanced online learners or take a more comprehensive, linear approach for those who still lack basic knowledge. Also, an online learner's history reveals that they prefer concrete eLearning activities, then, the system automatically adjusts their eLearning course map to recommendations that build related talents and 
abilities. [31] Proposed a framework for automatic online personalization through the recommendation process using artificial neural network techniques on different datasets.

Deep learning supports better resource allocation of online resources. So that online learners receive the exact online resources they require to fill gaps and achieve their learning goals. Also, it will allow the developers to spend less time analyzing graphs and LMS metrics, and more time developing powerful eLearning content.

Deep learning can automate the scheduling coursework for online learners or delivering online resources based on their eLearning assessment results or simulation performance. In the near future, it is possible to automatically generate unique eLearning course maps for every online learner who enrolls in the eLearning course.

Deep learning should improve eLearning return on investment (ROI) by providing less online training time and greater personalization translates into a broader profit margin. Deep learning models disclose hidden online training gaps to address the inadequacies and neglect other areas of the online training program that are no longer relevant and aren't resonating with online learners.

With the help of deep learning, online learners receive an individualized experience instead of a generic eLearning course that focus on irrelevant topics. So, the learners get motivated to engage with the eLearning content and reach their potential. In the future, a private virtual tutor may offer coursework which is necessary for the learners.

Deep learning system offers a comprehensive overview of big data and uses it to predict the outcome. For instance, online assessment results or survey findings. It can make peer-to-peer interactions more productive. For example, match mentors to online learners who can benefit from their specific skills or past experiences.

Apart from the quality of learning, deep learning presents an extremely valuable solution for training in industries with a high rate of dynamism with adaptive learning environments. Companies that need to update their course material on a continuous basis will benefit from deep learning as it can accurately predict how course material needs to improve and change. Adaptive learning technologies would give rise to completely personalized environments with content that not only changes but are created based on the individual needs of the learner. [19] Used Artificial Neural networks for generating adaptive lessons, their work showed the usefulness of the techniques based on some training, which is considered the main drawback of classical methods.

Deep learning environments can also analyze data across all personalized training instances intelligently, to recommend improvements and highlight inefficiencies that would not be possible otherwise. There is the benefit of using deep learning for translating content into other languages.

We have a responsibility to actively engage in the decision-making process, safeguard the practice and seek diversity of input for guidelines in the eLearning environment development, which will optimize the probable of personalized and adaptive learning to improve learning and performance outcomes of the learners. 


\section{Conclusion}

The use of technology to deliver learning has become a trend in the industry and has been termed, eLearning. It is necessary today for being an active learning perspective and engaging the students with the learning resources, deeply participating in the class and collaborating with each other and the teacher, rather than listening and memorizing. The students need to be motivated to demonstrate a process by simulation, analyzing an argument, or applying a concept to a real-world situation. Therefore, the care in organizing materials in the eLearning platform is essential. To improve existing e-learning applications, smart deep learning environments should, however, to provide personal services to help a learner use, manage, and interact with the learning system. Several studies have investigated the use of virtual and intelligent tutoring techniques, such as personalized learning interfaces and adaptive learning. These efforts have generally highlighted technology development but had little concern for effective instruction or pedagogy to enhance learning performance. Deep learning can apply user-centered design principles, to create new content, to know the target learners and design accordingly based on their needs and personalization of learning will exponentially improve as the learners acquire knowledge and process information and deploy learning best suited to learners.

\section{$7 \quad$ Acknowledgement}

We acknowledge King Khalid University for providing the opportunity to initiate this paper work.

\section{$8 \quad$ References}

[1] A. Fayyoumi, H. Mohammad, and H. Faris. (2013). Mobile based learning and examination: Students and instructors' perceptions from different Arab countries. Journal of Software Engineering and Applications, 6(12): 662. https://doi.org/10.4236/jsea.2013.612079

[2] A. Graves, R. Mohamed, and G. Hinton. (2013). Speech Recognition with Deep Recurrent Neural Networks. Acoustics, Speech and Signal Processing, IEEE International Conference. pp.6645 - 6649. https://doi.org/10.1109/icassp.2013.6638947

[3] A. Krizhevsky, I. Sutskever, and G. E. Hinton. (2012). Imagenet classification with deep convolutional neural networks. Advances in neural information processing systems. pp. 1097-1105. https://doi.org/10.1145/3065386

[4] AIaaS. https://www.bmc.com/blogs/ai-as-a-service-aiaas/

[5] Amazon Web Services. https://aws.amazon.com/machine-learning/

[6] Apache Singa. http://singa.apache.org/en/index.html.

[7] Britain, S., and Liber, O. (2004). A Framework for the Pedagogical Evaluation of Virtual Learning Environments. Report 41, JISC Technology Applications Programme. Available from: http://www.cetis.ac.uk/memt)ers/pedagogy/files/4thMeet_framework/VLEfullReport

[8] Caffe. (2018). http://caffe.berkeleyvision.org/

[9] Chapman, B. (2010). How Long Does It Take to Create Learning? [Research Study]. Available from: www.chapmanalliance.com 
[10] Colace Francesco, Santo, Massimo De and Greco, Luca. (2014). E-Learning and Personalized Learning Path: A Proposal Based on the Adaptive Educational Hypermedia System. International Journal of Emerging Technologies in Learning (iJET), 9 (2): 9-16. https://doi.org/10.3991/ijet.v9i2.3211

[11] Clark, R. C., \& Mayer, R. E. (2003). E-Learning and the science of education: Established strategies for consumers and designers of machine learning systems. San Francisco, CA: Jossey-Bass/Pfeiffer.

[12] Collis, B. (1997). Tele-learning in a digital world: The future of distance learning. London: International Thomson Computer Press.

[13] ConvNet, https://github.com/sdemyanov/ConvNet, last accessed 2019/05/15.

[14] Deep learning in Oncology. (2013) Available from: https://www.techemergence.com/ deep-learning-in-oncology/

[15] Deeplearning4j, https://deeplearning4j.org/, last accessed 2019/05/15.

[16] Ebner, M. (2007). E-Learning $2.0=$ e-Learning 1.0+ Web 2.0?” In.Conf. on Availability, Reliability and Security. IEEE ARES, 1235-1239.

[17] Edelstein, H.A. (1999). Introduction to Data Mining and Knowledge Discovery. 3rd Edition, Crows Corporation, Potomac.

[18] G. E. Dahl, D. Yu, L. Deng, and A. Acero. (2012). Context-dependent pre-trained deep neural networks for large-vocabulary speech recognition. Int. Conf. IEEE Acoustics, Speech and Signal Process. 20 (1): 30-42. https://doi.org/10.1109/tasl.2011.2134090

[19] Hassina Seridi, Toufik Sari and Mokhtar Sellami. (2006). Adaptive Instructional Planning using Neural Networks in Intelligent Learning Systems, The International Arab Journal of Information Technology, 3 (3): 183-192.

[20] Hu, Shanfeng et al. (2018). A Dual-Stream Recurrent Neural Network for Student Feedback Prediction using Kinect. In: SKIMA, Cambodia. https://doi.org/10.1109/skima.2018. $\underline{8631537}$

[21] IBM's Watson. https://www.ibm.com/watson/

[22] Jenkins, M., Browne, T., and Armitage, S. (2001). Management and implementation of Virtual Learning Environments. Report. UCISA. Available from: https://www.ucisa.ac.uk/

[23] Khan B.H. (1998). Web-based Instruction (WBI): An Introduction. Educational Media International, 35(2):63-71. https://doi.org/10.1080/0952398980350202

[24] Khan B.H. (2000). Discussion of resources and attributes of the web for the creation of meaningful learning environments. Cyber Psychology \& Behavior,3(1):17-23.

[25] L. Deng, G. Hinton, and B. Kingsbury. (2013). New types of deep neural network learning for speech recognition and related applications: An overview, in Proc. Int. Conf. IEEE Acoustics, Speech and Signal Process., Vancouver, BC, Canada, pp.8599-8603. https://doi.org/10.1109/icassp.2013.6639344

[26] Lecun Y, Bottou L, Bengio Y, et al. (1998). Gradient-based learning applied to document recognition. In: Proc IEEE Inst Electr Electron Eng 86: 2278-324. https://doi.org/10.1109/ $\underline{5.726791}$

[27] Microsoft Azure. https://azure.microsoft.com/en-us/overview/ai-platform/

[28] MXNet. http://mxnet.incubator.apache.org.

[29] Patrick McClure and Nikolaus K. (2016). Representational Distance Learning for Deep Neural Networks. Front Comput Neurosci, 10: 131. https://doi.org/10.3389/fncom.2016. $\underline{00131}$

[30] Pahl, C. (2003). Managing evolution and change in web-based teaching and learning environments. Computers in Education, 40.99-114. https://doi.org/10.1016/s0360-1315(02)00 $\underline{100-8}$ 
[31] P. Ralph and J. Parsons. (2006). A Framework for Automatic Online Personalization, Proc. 39th Ann. Hawaii Int'l Conf. System Sciences. https://doi.org/10.1109/hicss.2006.10

[32] Rosenberg M. J. (2002). E-Learning: Strategies for Delivering Knowledge in the Digital Age, New York, NY, USA: McGraw-Hill, Inc.

[33] Ruhi Sarikaya, Geoffrey E. Hinton, and Anoop Deoras. (2014). Application of DBN for natural language understanding.IEEE/ACM Trans. Audio, Speech and Lang. Proc. 22(4):778-784. https://doi.org/10.1109/taslp.2014.2303296

[34] Sayan Guha (2018). AI Chatbots In eLearning: Trends Embracing across Digital Landscape. https://elearningindustry.com/ai-chatbots-in-elearning-trends-digital

[35] Science Daily. (2016). Artificial intelligence course creates AI teaching assistant. Available from: https://www.sciencedaily.com/releases/2016/05/160509101930.htm.

[36] Shen Xiaoxuan, et. al. (2016). Automatic Recommendation Technology for Learning Resources with Convolutional Neural Network. 30-34.

[37] Sun, Ai., Li, Ying-Jian.,Huang, Yueh-Min., and Li, Qiong. (2017). Using Facial Expression to Detect Emotion in E-learning System: A Deep Learning Method. 10.1007/978-3319-71084-6_52.

[38] Tensorflow. https://www.tensorflow.org

[39] Wang, M., Vogel, D., and Ran, W. (2011). Creating a performance-oriented e-learning environment: A design science approach. Information and Management, 48(7): 260-269. https://doi.org/10.1016/j.im.2011.06.003

[40] Y. Shen, X. He, J. Gao, L. Deng, and G. Mesnil. (2014). Learning semantic representations using convolutional neural networks for web search, Proceedings of the companion publication of the 23rd international conference on World wide web companion, pp.373374. https://doi.org/10.1145/2567948.2577348

[41] Zachary C Lipton, David C. Kale, Charles Elkan, Randall Wetzel. (2016). Learning to Diagnose with LSTM Recurrent Neural Networks. International Conference on Learning Representations (ICLR).

\section{Authors}

Dr. Anandhavalli Muniasamy completed $\mathrm{PhD}$ from Sikkim Manipal University of Computer Science Engineering, India. Her areas of interest are data mining, machine learning and soft computing. She has published more than 25 research publications in highly reputed international journals with the total citations around 170 . She had attended and presented in more than 18 national \& international conferences. She served as a principal investigator for the funded project from All India Council for Technical Education (AICTE) and served as a manuscript reviewer for renowned international journals for past 10 years, and $\mathrm{PhD}$ thesis reviewer for several universities. She has been invited to give guest lectures and serving as editorial board member for a few journals and conferences. She is member of the International Association of Engineers, IEEE - Western Saudi Arabic Section, Computer Society of India and International Association of Computer Science and Information Technology. Currently, she is an associate Professor at the College of Computer Science, King Khalid University, Abha, Kingdom of Saudi Arabia. https://orcid.org/0000-0001-8940-3954 anandhavalli.dr@gmail.com

Dr. Areej Alasiry is an assistant professor at the College of Computer Science, King Khalid University and currently hold the position of the college Vice Dean for 
Graduate Studies and Scientific Research. Areej Alasiry has a PhD in Computer Science and Information Systems from Birkbeck College, University of London, UK. https://orcid.org/0000-0003-4088-5942.areej.alasiry@kku.edu.sa

Article submitted 2019-08-02. Resubmitted 2019-09-14. Final acceptance 2019-09-21. Final version published as submitted by the authors. 